\title{
Central post stroke pain: What are the new insights?
}

\author{
I Putu Eka Widyadharma', Clarissa Tertia', IA Sri Wijayanti' ${ }^{1}$, Jimmy FA Barus ${ }^{2}$ \\ ${ }^{1}$ Department of Neurology, Faculty of Medicine, Udayana University, Sanglah Hospital, Bali, Indonesia \\ ${ }^{2}$ Department of Neurology, Faculty of Medicine, Atmajaya University, Jakarta, Indonesia
}

\begin{abstract}
Stroke causes disability with high morbidity and mortality in the world, causes a variety of disabilities and symptoms including disturbances in motor function, sensory, and cognitive. Sensory disability in post stroke patient can be categorized into two group; the one is stimulated by peripheral mechanism which we often called post stroke pain, and another one is stimulated by central mechanism which we called central post stroke pain (CPSP). Pain after stroke is usually underdiagnosed and poorly understood. In 1906, CPSP was called the thalamus pain syndrome by Dejerine and Roussy, but then it is known that CPSP can also developed in extra-thalamic stroke lesion. The prevalence of CPSP is approximately $1-12 \%$ in all around the world. CPSP occurs in one-third of overall post-stroke pain cases. In most cases, the onset of CPSP is within 1 month and then the incidence decreases with time. The most common manifestations are allodynia and dysesthesia. The pathophysiology itself remains clearly unknown in detail. Various theories such as central sensitization, disinhibition of medial thalamus, and central imbalance theory thought to be contribute in CPSP pathophysiology. This complexity make CPSP very difficult to manage. Some pharmacotherapies and non-pharmacotherapies have been studied to relieve pain in order to improve the quality life of CPSP sufferers. The aim of this article is to discuss the general view of central post stroke pain to increase the understanding and awareness of health giver in order to give sooner and better management for patient that can affect prognosis of the patient.
\end{abstract}

Keywords: stroke, central post stroke pain, central mechanism

\author{
Abbreviations \\ CPSP - central post stroke pain \\ AIDS - acquired immunodeficiency syndrome \\ IASP - International Association for the Study of Pain \\ NMDA - N-methyl-d-aspartate \\ TMS - transcranial magnetic stimulation \\ DN4 questionnaire - douleur neuropathique 4 questions \\ LANSS - Leeds assessment of neuropathic symptoms and signs
}

\section{INTRODUCTION}

Stroke causes disability with high morbidity and mortality in the world, especially in low and middle income countries [1-3]. Stroke can cause a variety of disabilities and symptoms including disturbances in motor function, sensory, and cognitive. Sensory deficits due to stroke usually occur when there are lesions in the thalamus region,
rTMS - repetitive transcranial magnetic stimulation MCS - motor cortex stimulation

DBS - deep brain stimulation

PVG - periventricular gray

PAG - periaqueductal gray

TENS - transcutaneous electrical nerve stimulation where the thalamus is for the sensory information. In 1906, this syndrome was called the thalamus pain syndrome by Dejerine and Roussy [2,4-9]. All strokes that cause lesions in the somatosensory tract such as the nucleus of ventrocaudal thalamus, lateral medulla can also cause CPSP. Therefore the term, 'thalamus pain syndrome' is replaced by central post stroke pain $[5,6,10,11]$. CPSP can occur in both ischemic and hemorrhagic strokes with no 
difference in prevalence. Epidemiological studies report that the prevalence of CPSP is around $1-12 \% \cdot[5,6,10]$.

Pain is a serious problem after a stroke. Post stroke pain is usually underdiagnosed and poorly understood. Post stroke pain is often recur because the respond partially to the therapy $[11,12]$. In general, there are 2 types of post-stroke pain, CPSP which stimulated by central mechanism and post stroke pain which stimulated by peripheral mechanism. Most common post stroke pain are persistent headache $(3.5-31 \%)$, painful spasticity, musculoskeletal pain, and shoulder pain (11-40\%) $[5,8,11,13,14]$. In central neuropathic pain there is only central sensitization mechanism, which is different from the mechanism in peripheral neuropathic pain that caused by both peripheral and central sensitization. CPSP occurs in one-third of overall post-stroke pain cases $[5,12,14]$.

Pain in the CPSP causes disruption in daily activities, emotional disturbances that lead to reduce of the quality of life of the sufferers. CPSP sufferers will experience functional decline, cognitive, sleep disturbance, poor social interaction, anxiety to depression that can lead to suicide $[1,2,5,12,15,16]$. CPSP is often overlooked because of too much focus on motor deficits that cause non-optimal management $[1,2,16]$. The purpose of this article is to discuss the general view of central post stroke pain to increase the understanding and awareness of health giver in order to give sooner and better management for patient that can affect prognosis of patient with CPSP.

\section{CENTRAL POST STROKE PAIN}

CPSP is a neuropathic pain that occurs chronically, more than 3 months, due to sequelae to the central nervous system due to a stroke affecting the central somatosensory system in accordance with lesion in the brain territory, without obvious nociceptive stimuli, psychogenic causes or peripheral neuropathic lesion [1,3,4,14,17-20]. Either ischemic stroke, hemorrhagic stroke, intracerebral hemorrhage or subarachnoid hemorrhage can cause CPSP [21]. Definition of central post stroke pain according to the International Association for the Study of Pain ( IASP) is pain that occurs as a direct consequence of a stroke when the lesion occurs due to damage to the somatosensory system
$[16,22]$. In patients with CPSP, there are allodynia, dysesthesia, and hyperalgesia which are characteristic of CPSP sufferers with about $85 \%$ of CPSP sufferers experienced allodynia and hyperesthesia $[1,5,8,11,13-15,18]$.

The onset of CPSP varies, which occur immediately after a stroke or many years later. Generally it occurs within 1 to 3 months after a stroke. In most cases, the onset of CPSP is within 1 month and then the incidence decreases with time; $36 \%$ occurs in 1 to 3 months, $12 \%$ in 3 to 6 months, $6 \%$ in 6 to 12 months, and $11 \%$ after 12 months $[11,18,22]$. Based on a study conducted by Seifert et al. in 2013, stated that CPSP can last for years and even for a lifetime [5]. Because of the variety of symptoms experienced by CPSP sufferers and variety of the onset, it is quite difficult to characterized CPSP. Moreover, CPSP can occur with other pain syndromes, that makes it more difficult to evaluate [11].

The risk factors for CPSP are young age, smoking, history of depression and severity of stroke. In a study conducted by Helsinki Stroke, young stroke sufferers were twice at risk to develop CPSP $[11,12]$. Localization of lesions of stroke patients is associated with the occurrence of CPSP. CPSP often occurs in stroke patients in the cortical area, thalamus and brain stem, especially in the sensory part of posterior, lateral and inferior thalamus, which is the termination site of the spinothalamic tract. This is because in all 3 areas there is a central sensory system pathway. Strokes in the basal ganglia area rarely cause CPSP [12,22,23].

CPSP is caused by a stroke in the central sensory system in which the spinothalamic tract is disturbed, thalamus, insula, and anterior cingulate cortex disinhibited and hyperexcitabilitated. These three areas have many interactions with the motor cortex and interfere the excitability of intra-cortical motor and cause cortical disinhibition by decreasing intra-cortical inhibition at low intervals $[3,16]$. Sensory abnormalities can be as an increased sensitivity to tactile stimuli and temperature. In about two-third of patients with CPSP, they have disturbances in touch and temperature, whereas vibration and proprioception disorders are less common [1]. Lesions in the nucleus ventroposterolateral of thalamus cause sensory disturbance in hemibody. In supratentorial lesions, the heaviest pain is felt in the extremities with deficit 
sensation in sharp or cold with predominance mediated by $A \delta$ fibers, whereas in infratentorial lesions pain is felt on the face with loss of thermal and heat pain which mediated by $\mathrm{C}$ fibers [1].

\section{PATHOPHYSIOLOGY OF CENTRAL POST STROKE PAIN}

The basic route mechanism of pain is by going through three processes, transduction which convert the first stimulus into an electrical stimulus and then transduced into chemical stimulus, transmission which transmit the stimulus along the neuronal tracts, and last the modulation. The stimulus then is being modulated to higher brain center regulation [24]. There are 2 pain component systems, namely the lateral pain system which is a sensory component and pain discrimination and the medial pain system which is a component of affective and motivational pain. In addition, there is also a role for periaqueductal gray matter (PAG) in modulating pain by transmitting descending signals that inhibit pain transmission by cells in the dorsal horn [25].

The pathophysiology of CPSP remains largely unknown in detail with various factors thought to be contribute to this. These factors include dysfunction of the spinothalamic tract which causes disturbances in thermal sensation especially cold, sensory deafferentiation that causes sensory loss, disinhibition on the medial side which causes hypersensitivity and central sensitization $[3,5,16,26]$. The pathophysiology underlying CPSP is believed to be related to the presence of hyperexcitability or the release of neurons located in the thalamus or cortex that are spontaneously damaged. Hyperactivity of thalamus is also supported by neurophysiological and neuroimaging studies that show increased cerebral blood flow in the thalamus $[6,26]$. Pain stimuli will be transmitted through the spinothalamic tract toward the lateral and medial thalamus. In the lateral thalamocortical pain pathway, this stimulus will be directed to the primary somatosensory cortex, secondary somatosensory cortex, and insula. The primary somatosensory cortex is associated with pain discrimination, while the secondary somatosensory cortex is associated with pain intensity, and insula which will process thermal and nociceptive information [5].

There are several neurotransmitters that play a role in pain mechanism. Tachykinins are the largest neuropeptides, including neurokinin A which binds to neurokinin type 2 (NK2) receptors, neurokinin $B$ which binds to neurokinin type 3 (NK3) receptors and SP which binds to neurokinin type 1 (NK1) receptors. These receptors will bind to G-protein coupled receptors which have an excitatory effect. Calcitonin gene-related peptide (CGRP) is produced in both central and peripheral nervous systems and located in the dorsal horn. CGRP causes excitatory release of calcium. In addition, prostaglandins can stimulate chemical mediators such as serotonin, bradykinin, SP and CGRP which can increase sensitization. Glutamate is also an excitatory neurotransmitter that plays a major role in modulating pain. Glutamate will bind to the NMDA receptor which can cause an increase in intracellular calcium. The increase in intracellular calcium causes central sensitization [24].

\section{DISINHIBITION THEORY}

Abnormal hyperactivity of neurons can be explained by the disinhibition theory expressed by Head and Holmes. In this theory it is said that in patients with chronic pain there are lesions on the lateral thalamus so that it interferes with the inhibition between the lateral and medial thalamus and this will cause pain. Then this theory was renewed; lesions in the lateral spinothalamicus that play a role in the cold-signaling pathway through the ventroposterolateral thalamus to the insula will cause disinhibition of the medial spinothalamicus which will project the stimulus to the anterior cingulate cortex through the nucleus dorsal medial thalamus which causes a burning sensation. This burning sensation is caused by damage to the transmission system from a cold sensation due to an imbalance between excitation and inhibition in the central nervous system $[5,6,11]$. Lesions in the lateral thalamus are the most common cause of CPSP $[5,26]$.

\section{CENTRAL IMBALANCE THEORY}

In this theory, allodynia is caused by the imbalance between the lateral and medial pain systems which associated with the sensory discrimination and affective-emotional aspect. This can be evidenced by an increase in insular activity in the lateral spinothalamicus and a decrease in the activity of the cingulate anterior cortex in the medial spinothalamicus which cause allodynia.[5] 


\section{CENTRAL SENSITIZATION THEORY}

This theory states that there is a decrease in inhibition and increased facilitation thereby increasing neuronal excitability. This is because there is an increase in excitatory amino acid activity which then activates N-methyl-d-aspartate (NMDA) receptors. Hyperexcitability in the cortex or thalamus will cause spontaneous pain $[5,10]$. The pathophysiology of this CPSP is seen in Figure 1.

\section{CLINICAL MANIFESTATIONS OF CENTRAL POST STROKE PAIN}

Clinical manifestations of CPSP are very difficult to describe clearly. Patients often state that there is a sensation of dysesthesia that is very painful and cannot be localized properly and can change dramatically from day to day. In addition, the duration, intensity and location of pain also vary greatly [5]. Symptoms can be induced either by non-painful stimulus (allodynia) or by painful stimulus (hyperalgesia) because of the increased of sensitivity to stimulation. This symptoms can be found from bedside sensory exam by the clinicians. However, the spontaneous symptoms can be examined objectively, because the symptoms are discovered from patient's description. The spontaneous symptoms can be felt continuously or only paroxysmal [11].

Sensory symptoms that are felt vary from mild, moderate to severe and can be a burning sensation, sharp pain (lancinating), pinprick, electrical shock, cold, tingling, sensation of laceration, throbbing sensation and so forth $[5,9,11,21]$. These sensory abnormalities can be grouped into abnormal pain (allodynia and hyperpathy), abnormal sensations (paresthesia and dysesthesia), changes in detection threshold (hypoesthesia and hyperesthesia), and changes in intensity (hyperesthesia and hyperalgia). Negative symptoms in patients with CPSP are decreased or loss of pinprick and temperature sensation, while positive symptoms consist of pain or discomfort that is stimulated by temperature, touch or pressure. Clinical manifestations of allodynia become one of the characteristics of pain felt by patients, which is about two-third of CPSP patient, while vibration and tactile disorders are rarely associated with CPSP [5,15,17,21].

In assessing pain in central post stroke pain, there are several assessments that can be used, including the pain detect question (PD-Q), the Leeds assessment of neuropathic symptoms and signs (LANSS), douleur neuropathic en 4 questions (DN4), VAS score, brief pain inventory (BPI), neuropathic pain symptoms inventory (NPSI), and neuropathic pain scale (NPS). All of these scales can be used in assessing neuropathic pain in CPSP patients $[14,15,22,27]$.

\section{CRITERIA DIAGNOSIS OF CENTRAL POST STROKE PAIN}

Neuropathic pain in patients with CPSP can vary depend on the location of the lesion. It can be either in the thalamus or extra thalamus; the area of the operculum-insula, parietal cortex, and later-

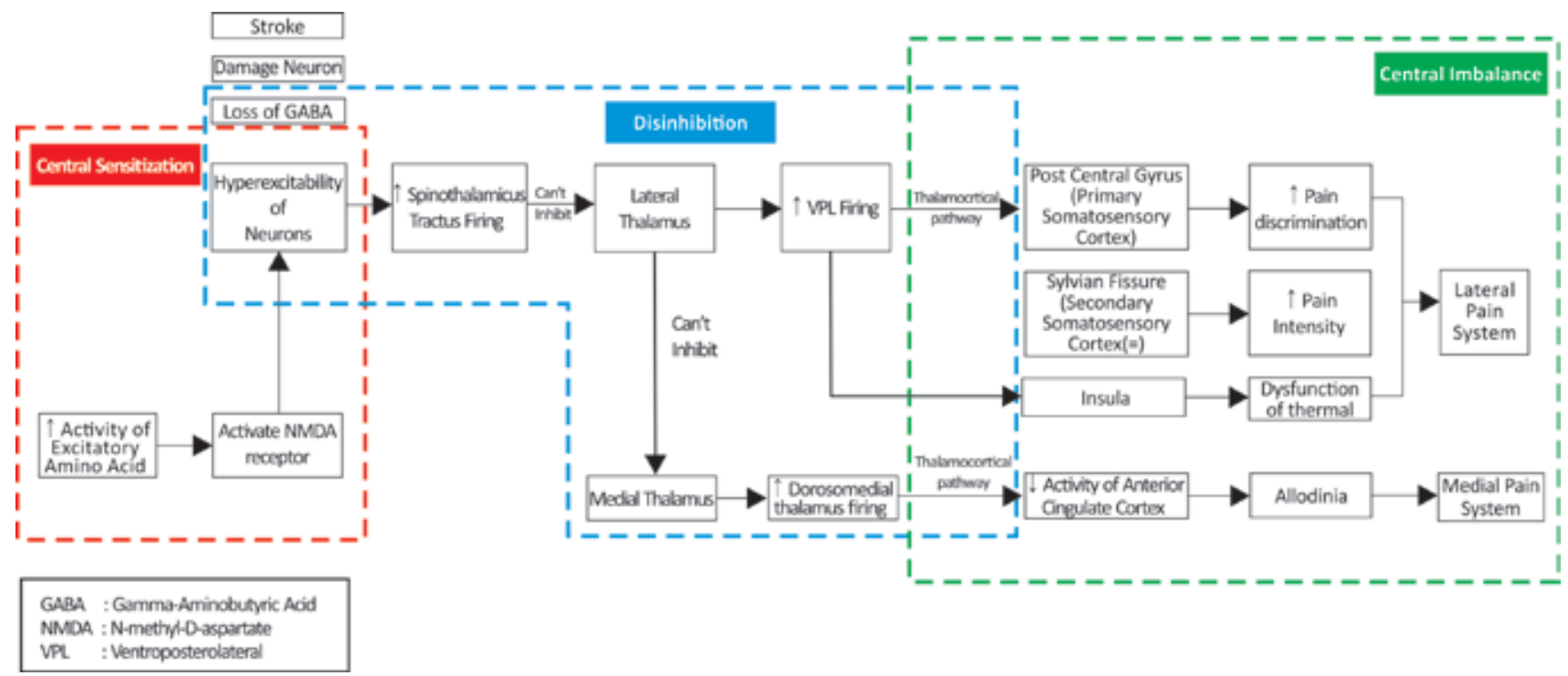

FIGURE 1. Pathophysiology of CPSP according to central sensitization theory 
al side medulla [16,21]. In 1999, Bowsher stated the criteria for diagnosis CPSP; certain CPSP (a history of stroke, subarachnoid hemorrhage, cerebral trauma, pain in the form of allodynia), highly probable CPSP (thermal sensation deficit and sensory discrimination), and probable CPSP if there was a history of burning sensation [5]. Klit et al. also provides criteria for a diagnosis of CPSP; possible CPSP if there is pain associated with neuroanatomy distribution, exclusion of other causes of pain, and a history of stroke, probable criteria if the above criteria are accompanied by pain which has been confirmed through clinical examination or associated with vascular lesions on imaging, and definite CPSP if all five points above have been met [13]. In addition, Hansen et al. in 2012 stated a simpler criteria such as pain occurs during a stroke or post stroke, the location of pain is related to the location of stroke and no cause of pain including pain in the shoulder joint and its surroundings. Seifert et al. in 2013 stated that the diagnosis of CPSP must be based on a combination of history, sensory examination using various types of somatosensory stimuli such as thermal, pressure, pinprick and vibration, and also the discovery of lesions in neuroimaging [5].

\section{MANAGEMENT OF CENTRAL POST STROKE PAIN}

According to the International Association for the Study of Pain Neuropathic Pain Special Interest Group, the European Federation of Neurological Societies, and the Canadian Pain Society, tricyclic antidepressant is the first-line therapy for patients with CPSP. In addition, the European Federation of Neurological Societies and the Canadian Pain Society also recommend anti-convulsants as first-line therapy [18]. Amitriptyline, a tricyclic antidepressant group, is considered to be the firstline therapy for CPSP because of its effectiveness in relieving pain that has been studied since 1989 . However, there are side effects of the use of amitipriline, such as constipation, dry mouth, sedation, orthostatic hypotension, urinary retention, cardiac arrhythmias, and decreased of seizure threshold $[4,5,11,16,21]$. However, in a study conducted by Mulla et al.; there was no significant effect in giving antidepressant tricyclic in CPSP patient with an increase in side effects 2 times greater than placebo $(95 \% \mathrm{CI}=1.15-3.49)[18]$.
In addition, anti-convulsant drugs that have been investigated for patients with CPSP such as carbamazepine, pregabalin, gabapentin, lamotrigine and levetiracetam [5]. In a study conducted by Mulla et al., anti-convulsant therapy did not significantly reduce pain intensity when compared with placebo with an increased risk of 1.6 times greater for side effects $(95 \% \mathrm{CI}=0.90-2.88)$. Administration of carbamazepine only reduce pain in the initial 3 weeks. Other anti-convulsant groups such as gabapentin have been proven effective and can be given to patients with other neuropathic pain such as diabetic neuropathy. Gabapentin has lesser side effects, safer and interacts less with other drugs. Gabapentin can be considered as second-line therapy [21]. In a study conducted by Kim et al. in 2010 , states that pregabalin can reduce pain significantly, but this is only found in the first 8 weeks of therapy. After 8 weeks, pregabalin did not give a significant difference in reducing pain when compared to placebo [4]. In contrast, in a double-blind study of 219 patients, pregabalin did not give significant results, but pregabalin can improve anxiety, sleep and quality of life, with minor side effects such as edema, weight gain, and dizziness [11].

In patients with CPSP who do not get a good response to pharmacological therapy can be given non-pharmacotherapy treatment such as repetitive transcranial magnetic stimulation (rTMS), motor cortex stimulation (MCS), deep brain stimulation (DBS), acupuncture, and mirror therapy $[5,18,21]$. In most studies it is reported that repetitive transcranial magnetic stimulation (rTMS) which acts in the primary motor cortex can decrease pain in CPSP [15]. rTMS is a non-invasive procedure and proven to be safe without severe side effects and effective in treating neuropathic pain. The mechanism of rTMS in neuropathic pain is associated with the activation of projection of inhibition of the motor cortex or pre motor to the sensory nucleus in the thalamus. In one study, a significant reduction in pain was found in patients with CPSP after rTMS $(p=0.025)[5,21]$. In a study, rTMS that given consecutively for 5 days showed a decrease in pain in CPSP sufferers but this is only temporary so it cannot be a single therapy [5].

MCS is an invasive neuromodulation technique that inhibits the thalamus and disinhibited PAG [10]. MCS not only changes activity in the thalamus, but also in the cingulate anterior cortex and 
also the anterior insula which play role in emotional function. MCS modulates the pain pathways associated with mood and emotions. MCS is effective in reducing pain temporarily in CPSP according to a study conducted by Tanei et al. in 2011. The efficacy of MCS depends on the accuracy of the location of the MCS and also the progamming of stimulation parameters [5,12]. Based on the study of Katayama et al., MCS need an intact corticospinal pathway in order to give significant effect. MCS does not provide meaningful results in CPSP patients with motor weaknesses especially moderate to severe degrees $[6,15]$.

Deep brain stimulation (DBS) is a neuromodulation procedure with insertion of electrodes to the target area in the brain; the periventricular gray (PVG) area, periaqueductal gray (PAG) and the ventroposterolateral thalamus nucleus area. DBS showed the effectiveness of therapy in $70 \%$ of CPSP sufferers. DBS can be done with a combination of MCS. In studies using DBS placed on periventricular gray, it can relieved allodynia in hemibody that lasted only 9 months and then the pain reappeared [5,6,10,11,21,27]. The use of MCS and DBS in CPSP sufferers did not give results as well as in patients with peripheral neuropathy pain [28]. Transcutaneous electrical nerve stimulation (TENS), acupuncture / apipuncture can reduce pain temporarily in patients with CPSP, but has not shown clear efficacy. In the RCT study by Cho et al., giving acupuncture for 3 weeks provided a significant reduction in pain through a VAS score $(p=0.009)$ but a larger sample was still needed to assess its efficacy $[5,8]$.

Literature explained that mirror therapy can also suppress proprioceptive inputs that are not ap-

Conflict of interest: none declared

Financial support: none declared

\section{REFERENCES}

1. Kumar B, Kalita J, Kumar G, Misra UK. Central poststroke pain: A review of pathophysiology and treatment. Anesth Analg. 2009;108(5):1645-57.

2. Bashir AH, Abdullahi A, Abba MA, Mukhtar NB. Central Poststroke Pain: Its Profile among Stroke Survivors in Kano, Nigeria. Behav Neurol. 2017;2017.

3. Tang SC, Lee LJH, Jeng JS, Hsieh ST, Chiang MC, Yeh SJ, et al. Pathophysiology of central poststroke pain motor cortex disinhibition and its clinical and sensory correlates. Stroke. 2019;50(10):2851-7.

4. Kim JS, Bashford G, Murphy TK, Martin A, Dror V, Cheung R. Safety and efficacy of pregabalin in patients with central post-stroke pain. Pain. 2011;152(5):1018-23.

5. Oh H, Seo W. Review Article A Comprehensive of Central Post-Stroke Pain. Pain Manag Nurs. 2015;16(5):1-15. propriate by inducing plasticity of sensorimotor neurons and visual information. The patient is given a mirror that can reflect a healthy limb so that it gives the perception that the affected limb looks healthy. Mirror therapy was first introduced in 1995 in a study that reported a reduction in phantom pain in an amputated arm, which has now been widely applied to motor healing after a stroke [26]. Based on these findings, the use of mirror therapy is expected to reduce the perception of pain. In a study conducted by Corbetta et al., mirror therapy can be done as an additional approach for CPSP because mirror therapy can change a patient's perception of pain [26].

\section{CONCLUSIONS}

CPSP is a complex disease that is difficult to treat. The purpose of treatment in CPSP is to reduce pain in order to improve the quality of life, because patients with CPSP will not be able to recover completely. A combination of psychological and medical interventions must be applied. In CPSP, there is an intra-cortical motor inhibition without changes in afferent inhibition indicating there is a disruption in the function of gamma-amino-butiric-acid in this pain syndrome. Patients will experience psychological disorders including decreased sleep quality, decreased social interaction, anxiety and depression. The onset of CPSP varies that it often causes late administration of therapy. Given aggressive and earlier therapy can affect the prognosis of patients with CPSP. Understanding and awareness of CPSP is very important in providing better management.

6. Morishita T, Inoue T. Brain stimulation therapy for central post-stroke pain from a perspective of interhemispheric neural network remodeling. Front Hum Neurosci. 2016;10:1-8.

7. De Vloo P, Morlion B, Van Loon J, Nuttin B. Animal models for central poststroke pain: A critical comprehensive review. Pain. 2017;158(1):17-29.

8. Singer J, Conigliaro A, Spina E, Law SW, Levine SR. Central poststroke pain: A systematic review. Int J Stroke. 2017; 12(4):343-55.

9. Park JM, Lee JS, Jeong JE, Lee YJ, Lee CK, Roh J Du, et al. Chronic Central Post-Stroke Pain Treated with Scalp Acupuncture and Traditional Korean Medicine: A Case Report. J Acupunct Res. 2019;36(4):277-81.

10. Akyuz G, Kuru P. Systematic review of central post stroke pain. Am J Phys Med Rehabil. 2016;95(8):618-27. 
11. Treister AK, Hatch MN, Cramer SC, Chang EY, Diego S, Beach L, et al. Demystifying post-stroke pain : from etiology to treatment. 2018;9(1):63-75.

12. Harrison RA, Field TS. Post stroke pain: Identification, assessment, and therapy. Cerebrovasc Dis. 2015;39(3-4):190-201.

13. Klit H, Finnerup NB, Andersen $G$, Jensen TS. Central poststroke pain: A population-based study. Pain. 2011;152(4):818-24.

14. Haroutounian S, Ford AL, Frey K, Nikolajsen L, Finnerup NB, Neiner $A$, et al. How central is central poststroke pain? The role of afferent input in poststroke neuropathic pain: A prospective, open-label pilot study. Pain. 2018;159(7):1317-24.

15. Goto T, Saitoh Y, Hashimoto N, Hirata M, Kishima H, Oshino S, et al. Diffusion tensor fiber tracking in patients with central post-stroke pain; correlation with efficacy of repetitive transcranial magnetic stimulation. Pain. 2008;140(3):509-18.

16. Sciupokas A. Central Post Stroke Pain: The Clinical-Anatomical Correlations from Pain Clinic Stories. Biomed J Sci Tech Res. 2018;11(4):8653-8.

17. Klit $H$, Hansen AP, Marcussen NS, Finnerup NB, Jensen TS. Early evoked pain or dysesthesia is a predictor of central poststroke pain. Pain. 2014;155(12):2699-706.

18. Mulla SM, Wang L, Khokhar R, Izhar Z, Agarwal A, Couban R, et al. Management of central poststroke pain: Systematic review of randomized controlled trials. Stroke. 2015;46(10):2853-60.

19. Shih HC, Kuan YH, Shyu BC. Targeting brain-derived neurotrophic factor in the medial thalamus for the treatment of central poststroke pain in a rodent model. Pain. 2017;158(7):1302-13.

20. Mizumoto J. Central Poststroke Pain with Wallenberg Syndrome. Am J Med. 2020;133(1):e11-2.
21. Flaster M, Meresh E, Rao M, Biller J. Central poststroke pain: Current diagnosis and treatment. Top Stroke Rehabil. 2013;20(2):116-23.

22. Vukojevic Z, Dominovic Kovacevic A, Peric S, Grgic S, Bjelica B, Basta I, et al. Frequency and features of the central poststroke pain. J Neurol Sci. 2018;391(2017):100-3.

23. Krause T, Asseyer S, Geisler F, Fiebach JB, Oeltjenbruns J, Kopf A, et al. Chronic sensory stroke with and without central pain is associated with bilaterally distributed sensory abnormalities as detected by quantitative sensory testing. Pain. 2016;157(1):194-202.

24. Yam MF, Loh YC, Tan CS, Khadijah Adam S, Abdul Manan N, Basir R. General Pathways of Pain Sensation and the Major Neurotransmitters Involved in Pain Regulation. Int J Mol Sci. 2018 Jul 24;19(8):2164.

25. Sayat ARG, Mestecky A, Hassanzadeh A. Assessment and treatment of central post-stroke pain: an overview. British Journal of Neuroscience Nursing 2019;15(Sup2).

26. Corbetta D, Sarasso E, Agosta F, Filippi M, Gatti R. Mirror therapy for an adult with central post-stroke pain: a case report. Arch Physiother. 2018;8(1):4-9.

27. Pickering AE, Thornton SR, Love-Jones SJ, Steeds C, Patel NK. Analgesia in conjunction with normalisation of thermal sensation following deep brain stimulation for central post-stroke pain. Pain. 2009;147(1-3):299-304.

28. Gopalakrishnan R, Burgess RC, Lempka SF, Gale JT, Floden DP, Machado AG. Pain anticipatory phenomena in patients with central poststroke pain: A magnetoencephalography study. J Neurophysiol. 2016;116(3):1387-95 